\title{
Investigating dietary acculturation and intake among US-born and Thailand/Laos-born Hmong-American children aged 9-18 years
}

\author{
Urvashi Mulasi-Pokhriyal $^{1}$, Chery Smith ${ }^{1, *}$ and Lisa Franzen-Castle ${ }^{2}$ \\ 'Department of Food Science and Nutrition, University of Minnesota, 225 FScN, 1334 Eckles Avenue, Saint \\ Paul, MN 55108-6099, USA: ${ }^{2}$ Panhandle Research and Extension Center, University of Nebraska-Lincoln, \\ Scottsbluff, NE, USA
}

Submitted 28 September 2010: Accepted 3 June 2011: First published online 2 August 2011

\begin{abstract}
Objective: The Hmong are a growing population of South-East Asian immigrants with increasing rates of obesity and diabetes, yet little is known about their dietary consumption patterns. The present study aimed to investigate the dietary intake of Hmong children and whether acculturation and/or time lived in the USA influences dietary intake, BMI and nutritional status.

Design: Two $24 \mathrm{~h}$ dietary recalls were collected on non-consecutive days using the multiple-pass interviewing method and were averaged. Heights and weights were measured, from which BMI was calculated. An acculturation score was computed. Setting: Schools, churches, Hmong organizations, and community centres.

Subjects: Three hundred and thirty-five Hmong children aged 9-18 years from Twin Cities, Minnesota, USA.

Results: Approximately half of our participants were either overweight or obese. US-born children were significantly heavier, taller, had a higher BMI, and in general consumed more energy, saturated fat and $\mathrm{Na}$ than those who were born in Thailand/Laos and were living in the USA for $<5$ years. Children who were more acculturated to US norms including language use, social connections and dietary habits had higher BMI-for-age and consumed significantly more saturated fat, trans fatty acids, $\mathrm{Na}$ and $\mathrm{Ca}$ compared with their less acculturated counterparts. Conclusions: Diets of most Hmong children appear below the recommendations for fibre, vitamins A, D and E, Ca, P, Mg and K, and are higher in fats, sugars and $\mathrm{Na}$. Living in an obesogenic US environment is a probable reason for poor dietary quality of Hmong and may be a contributing factor to the rising rates of obesity and diabetes in this population.
\end{abstract}

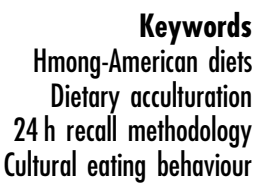

Keywords

Hmong-American diets $24 \mathrm{~h}$ recall methodology Cultural eating behaviour
According to the US Census Bureau, over 100 million US residents are now considered to be from a minority group $^{(1)}$. With a population of about 15 million $^{(2)}$, Asian Americans, including the Hmong, are one of the fastestgrowing minority populations in the USA. The Hmong, a South-East Asian group originally from Laos, secretly assisted the US military and Central Intelligence Agency during the Vietnamese Conflict (1963-1975) ${ }^{(3)}$. After the Conflict, communists targeted Hmong because of their help to the USA and many Hmong suffered hardships including genocide, poverty, excessive labour, depression and food insecurity, and consequently fled Laos and settled in refugee camps in Thailand ${ }^{(4-8)}$. Conditions of refugee camps varied, but poverty and food insecurity were common, leading to Hmong migration to countries such as Australia, France and the USA ${ }^{(4,6,8)}$. Today it is estimated that about 200000 Hmong live in the USA ${ }^{(2)}$.
This number is expected to grow because Hmong tend to have larger families; the average family size of Hmong Americans is 6.51 people, much higher compared with $3 \cdot 14$ people in the average American family ${ }^{(9)}$.

Immigration to the USA has introduced the once physically active Hmong to an obesogenic American environment. Franzen and Smith ${ }^{(4)}$ found that after immigrating to the USA, environmental changes and increased acculturation to American dietary habits have negatively impacted the weight and health status of this population. Increased rates of obesity and obesity-related conditions have been noted among the Hmong ${ }^{(4,5,10-14)}$. In a sample of adult Hmong refugees ( $n$ 448, aged $>20$ years), Culhane-Pera et $a l^{(12)}$ found that $33 \%$ of the sample was overweight and 15\% obese. Further, diabetes rates also seem to be rising in this group ${ }^{(15)}$ and among Hmong adults in the USA, the rate of diabetes is estimated to be 
twenty times higher than that of Hmong adults in Thailand $^{(16)}$. Her and Mundt ${ }^{(17)}$ found among Wisconsin Hmong adults ( $n$ 144) that $41 \%$ had casual capillary blood glucose levels $\geq 140 \mathrm{mg} / \mathrm{dl}$, considered a positive screen test for diabetes. Among children, the Centers for Disease Control and Prevention (CDC) ${ }^{(18)}$ estimated that the rate of obesity-dependent type 2 diabetes is greater than type 1 diabetes among Asian/Pacific Islanders younger than 20 years of age.

The US environment appears to have influenced Hmong dietary and food-related habits. In Laos, traditional Hmong diets were higher in complex carbohydrates, boiled vegetables and seasonal fruits, and water and vegetable/ meat broths were the usual beverages of choice ${ }^{(4,13)}$. Additionally, desserts were rarely consumed and snacking was atypical $^{(4)}$. After immigration to the USA, an increased consumption of saturated fats, sugars, refined grains and salt has been noted among the Hmong ${ }^{(4,5,13)}$.

Little is known about the dietary intake of Hmong adults or children at the nutrient level. In focus group discussions, dietary behaviours and acculturation among Hmong children (9-18 years) were explored, but individual dietary intake was not assessed ${ }^{(5)}$. Vue and Reicks ${ }^{(19)}$ assessed intake of Ca-rich foods and beverages among 10-13-year-old Hmong girls through questionnaires and parental interviews but did not collect dietary data on other nutrients/food groups.

To our knowledge, comprehensive dietary intake and BMI status for school-aged Hmong children has not been studied and research investigating Hmong dietary practices, current nutritional status and post-migration impact on dietary acculturation is also very limited. Knowing that Hmong are a growing ethnic group in the USA and with increasing rates of obesity and diabetes in this group, it is necessary to investigate the dietary consumption patterns of Hmong children so that appropriate and timely interventions may be planned. Therefore, the purposes of the present study were to: (i) investigate whether time lived in the USA and the degree of acculturation impact the quality and quantity of diet; and (ii) assess differences in food consumption patterns by food groups and nutrient intakes for Hmong children born in the USA compared with those recently immigrated from Thailand or Laos. To the best of our knowledge, ours is the first study which assesses dietary intake among Hmong specifically from an acculturation perspective, incorporating detailed quantitative methodology.

\section{Experimental methods}

\section{Participants}

Three hundred and thirty-five Hmong children (9-18 years) living in Minneapolis/Saint Paul, Minnesota, participated in the present study. Some children were born and/or raised in the USA (born-US) and were either 9-13 years old
( $n$ 144) or 14-18 years old ( $n$ 156). A small number were born and/or raised in Thailand/Laos and had been in the USA for $<5$ years (born-T/L) and were either 9-13 years old ( $n$ 21) or 14-18 years old ( $n$ 14). Hmong organizations and key informants assisted in recruitment efforts and in total seventeen different sites were visited to maximize diversity within the sample. Children were recruited through activitybased organizations (54\%), Hmong schools (26\%), churches (12\%), and via advertisement in the local Hmong newspaper ( $8 \%)$. Informed parental consent/child assent was obtained and the University of Minnesota's Institutional Review Board approved this study.

\section{Dietary recall metbodology}

Two $24 \mathrm{~h}$ dietary recalls were collected by trained researchers on non-consecutive days $(30 \%$ of the recalls included a weekend day) and averaged in order to better describe each child's usual intake of food and nutrients. While interviewing children, a four-stage, multiple-pass technique was used ${ }^{(20)}$. During stage 1 , a complete list of all foods and beverages consumed by the child was obtained. Stage 2 involved a detailed description of each food and beverage consumed, and cooking methods and food brand names were also asked. An estimated amount of each food and beverage item consumed was obtained in stage 3. Lastly, in stage 4, the recall was reviewed by the researcher with the child to ensure that all items, including dietary supplementation, had been recorded. While the $24 \mathrm{~h}$ dietary recall has limitations for individual assessment, it can be useful in comparing groups ${ }^{(21)}$. To evaluate dietary assessment methods used among 5-18-year-olds, McPherson et al. ${ }^{(22)}$ examined thirty-eight validity and nine reliability studies. Correlations between the dietary method and the validation standard were higher for $24 \mathrm{~h}$ recall and food record methodologies than for FFQ. Furthermore, Frank ${ }^{(23)}$ suggested the $24 \mathrm{~h}$ recall method to be a reliable tool for ages 9 years and above.

In addition to using the multiple-pass interviewing technique, memory prompts such as colourful food models, measuring cups and food pictures were used as aids to reduce error, improve the quality and accuracy of the intake, and to provide children with models to estimate portion sizes. Food models included foods consumed in both Hmong and American cultures such as rice, stir-fries, soups, bread, milk, pizza and cereal.

\section{Acculturation score}

Acculturation level was assessed using ten questions asking about language use, social connections and overall dietary habits. The acculturation tool used in the present project was previously created by Marin et al. ${ }^{(24)}$ for Hispanics, and has reliability/validity coefficients comparable to other published acculturation tools ${ }^{(5)}$. This tool has successfully been used with Hmong adults ${ }^{(4)}$. Prior to using the tool with a larger group of children, it was pilottested and assessed for ease of use and readability with 
twenty-two Hmong children; these children reported no difficulties with the tool (Flesch Reading Ease score was $84 \cdot 3$ (easy to read) and Flesch-Kincaid Grade Level was 4th). To measure the reliability of the acculturation tool, children completed the same acculturation assessment at two different times. Paired-samples $t$ tests were then computed to determine whether there were significant differences in children's responses between the two assessments; no significant differences were found, suggesting that this was an appropriate tool for children. Acculturation score was determined by summing the responses to individual questions and a higher score indicated more acculturation to US norms. Sample questions were: (i) 'What language do you usually speak at home?' (ii) 'Your closest friends are?' (iii) 'I eat ___ foods'. Possible responses to these questions were: (i) 'only Hmong'; (ii) 'more Hmong than American'; (iii) 'both Hmong and American'; (iv) 'more American than Hmong'; and (v) 'only American'.

\section{Antbropometric measures}

Heights and weights were measured using standard procedures $^{(25)}$ without outer heavier clothing and shoes. BMI was calculated as weight in kilograms divided by the square of height in metres, and plotted on the CDC BMIfor-age gender specific growth charts to obtain a percentile, which ranks underweight children as $<5$ th percentile, healthy weight as $\geq 5$ th to $<85$ th percentile, overweight as $\geq 85$ th to $<95$ th percentile and obese children as $\geq 95$ th percentile ${ }^{(26)}$. Stature rankings were $<5$ th percentile for short, $\geq 5$ th to $<85$ th percentile for average, and $\geq 85$ th percentile for tall children.

\section{Data analysis}

Data were first checked for normality and analysed using the Predictive Analytics SoftWare (PASW) statistical software package version 17 (formerly SPSS; IBM Corporation, Armonk, NY, USA). Descriptive statistics computed means, standard deviations and frequencies (Table 1). The $24 \mathrm{~h}$ dietary recalls were analysed using the ESHA Food Processor ${ }^{\circledR}$ SQL Software version $10 \cdot 4 \cdot 0$ (ESHA Research, Salem, OR, USA), which computed nutrient and MyPyramid intakes. The 2010 Dietary Reference Intakes (DRI) were used as a reference for each nutrient recommended within a specific age group (9-13 years and 14-18 years) and gender ${ }^{(27)}$ (Tables 2 and 3). MyPyramid guidelines were used to compute servings of grains, vegetables, fruits, milk, meat and beans, and fats, oils and sweets $^{(28,29)}$. A serving of fat was the number of grams in 1 tbsp of fat for butter, margarine, oils and shortening $^{(28,29)}$. For meats, an additional fat serving was reported as a multiple of the fat standard for the specific meat, and for milk products and mixed foods, an additional fat serving was reported as a multiple of $12 \cdot 8 \mathrm{~g}$, the weight of 1 tbsp of shortening ${ }^{(29)}$. A serving of sugar was defined as the number of grams in 1 tsp of sugar $(4 \mathrm{~g})^{(28,29)}$.

Table 1 Sample characteristics of Hmong children aged 9-18 years from Twin Cities, Minnesota, USA

\begin{tabular}{|c|c|c|c|c|c|c|c|c|}
\hline & \multicolumn{4}{|c|}{ Males } & \multicolumn{4}{|c|}{ Females } \\
\hline & \multicolumn{2}{|c|}{$\begin{array}{c}9-13 \text { years } \\
(n \text { 82) }\end{array}$} & \multicolumn{2}{|c|}{$\begin{array}{c}\text { 14-18 years } \\
(n 68)\end{array}$} & \multicolumn{2}{|c|}{$\begin{array}{c}9-13 \text { years } \\
(n \text { 83) }\end{array}$} & \multicolumn{2}{|c|}{$\begin{array}{c}14-18 \text { years } \\
(n 102)\end{array}$} \\
\hline & Mean & $\mathrm{SD}$ & Mean & SD & Mean & SD & Mean & SD \\
\hline Age (years) & $11 \cdot 4$ & $1 \cdot 3$ & $15 \cdot 7$ & $1 \cdot 4$ & $11 \cdot 2$ & $1 \cdot 3$ & $15 \cdot 8$ & $1 \cdot 2$ \\
\hline \multicolumn{9}{|l|}{ Anthropometric measurements } \\
\hline Weight $(\mathrm{kg})$ & $48 \cdot 3$ & 18 & $68 \cdot 1$ & 18 & $44 \cdot 8$ & 14 & $58 \cdot 6$ & 13 \\
\hline Height (cm) & 145 & 11 & 163 & 7 & 144 & 9 & 153 & 5 \\
\hline \multirow[t]{2}{*}{ BMI $\left(\mathrm{kg} / \mathrm{m}^{2}\right)$} & $22 \cdot 4$ & 5 & $25 \cdot 5$ & 6 & $21 \cdot 3$ & 5 & $25 \cdot 0$ & 6 \\
\hline & $n$ & $\%$ & $n$ & $\%$ & $n$ & $\%$ & $n$ & $\%$ \\
\hline \multicolumn{9}{|l|}{ Grade level } \\
\hline Middle school & 50 & 61 & 8 & 12 & 46 & 55 & 9 & 9 \\
\hline High school & - & - & 59 & 87 & - & - & 92 & 90 \\
\hline Post-secondary & - & - & 1 & 1 & - & - & 1 & 1 \\
\hline \multicolumn{9}{|l|}{ Birth location } \\
\hline Born-US & 73 & 89 & 60 & 88 & 71 & 85 & 96 & 94 \\
\hline Born-T/L & 9 & 11 & 8 & 12 & 12 & 14 & 6 & 6 \\
\hline \multicolumn{9}{|l|}{ Height-for-age percentile } \\
\hline$<5$ th percentile (short) & 5 & 6 & 20 & 29 & 9 & 11 & 44 & 43 \\
\hline$\geq 5$ th to $<85$ th percentile (average) & 69 & 84 & 47 & 69 & 68 & 82 & 58 & 57 \\
\hline$\geq 85$ th percentile (tall) & 8 & 10 & 1 & 1 & 6 & 7 & - & - \\
\hline \multicolumn{9}{|l|}{ BMI percentile } \\
\hline$\geq 5$ th to $<85$ th percentile (healthy weight) & 40 & 49 & 31 & 46 & 51 & 61 & 57 & 56 \\
\hline$\geq 85$ th to $<95$ th percentile (overweight) & 12 & 15 & 10 & 15 & 14 & 17 & 18 & 18 \\
\hline$\geq 95$ th percentile (obese) & 31 & 38 & 26 & 38 & 18 & 22 & 27 & 26 \\
\hline
\end{tabular}

Born-T/L, born and/or raised in Thailand or Laos and had been living in the USA for $<5$ years; born-US, born in the USA.

Dashes indicate no values. 
Table 2 Dietary intakes by birth location for 9-13-year-old Hmong children from Twin Cities, Minnesota, USA, compared with DRI

\begin{tabular}{|c|c|c|c|c|c|c|c|c|c|c|c|c|c|c|}
\hline & \multicolumn{7}{|c|}{ Males (9-13 years; $n$ 82) } & \multicolumn{7}{|c|}{ Females (9-13 years; $n$ 83) } \\
\hline & \multirow[b]{2}{*}{ DRI } & \multicolumn{3}{|c|}{ Born-T/L } & \multicolumn{3}{|c|}{ Born-US } & \multirow[b]{2}{*}{ DRI } & \multicolumn{3}{|c|}{ Born-T/L } & \multicolumn{3}{|c|}{ Born-US } \\
\hline & & Mean & SD & PBD & Mean & SD & PBD & & Mean & SD & PBD & Mean & SD & PBD \\
\hline Acculturation score & - & $19 \cdot 5^{\star}$ & $5 \cdot 5$ & - & $28 \cdot 1$ & $5 \cdot 3$ & - & - & $20 \cdot 4^{*}$ & $4 \cdot 8$ & - & $28 \cdot 5$ & $4 \cdot 5$ & - \\
\hline Energy $(\mathrm{kJ} / \mathrm{d})$ & - & $5667^{\star}$ & 1255 & - & 7164 & 2379 & - & - & 6429 & 1545 & - & 6376 & 2045 & - \\
\hline Energy $(\mathrm{kcal} / \mathrm{d})$ & - & $1354 \cdot 5^{\star}$ & $300 \cdot 0$ & - & $1712 \cdot 3$ & $568 \cdot 6$ & - & - & $1536 \cdot 5$ & $369 \cdot 3$ & - & 1523.9 & $488 \cdot 8$ & - \\
\hline Carbohydrates (g/d) & 130 & $168 \cdot 5^{\star}$ & $35 \cdot 3$ & $11 \cdot 0$ & $213 \cdot 7$ & $74 \cdot 1$ & $9 \cdot 6$ & 130 & $197 \cdot 6$ & $42 \cdot 0$ & $8 \cdot 3$ & $198 \cdot 4$ & $72 \cdot 1$ & $18 \cdot 3$ \\
\hline Protein $(\mathrm{g} / \mathrm{d})$ & 34 & $72 \cdot 1$ & $25 \cdot 1$ & 0.0 & $78 \cdot 7$ & $29 \cdot 7$ & $4 \cdot 1$ & 34 & $73 \cdot 0$ & $24 \cdot 5$ & 0.0 & $66 \cdot 9$ & $26 \cdot 3$ & $7 \cdot 0$ \\
\hline Fat $(g / d)$ & - & $43 \cdot 4^{*}$ & $16 \cdot 2$ & - & $60 \cdot 7$ & $27 \cdot 6$ & - & - & $50 \cdot 8$ & $20 \cdot 0$ & - & $51 \cdot 7$ & $23 \cdot 2$ & - \\
\hline Saturated fat $(\mathrm{g} / \mathrm{d})$ & - & $14 \cdot 7^{\star}$ & $5 \cdot 1$ & - & $21 \cdot 2$ & $9 \cdot 7$ & - & - & $17 \cdot 8$ & $7 \cdot 3$ & - & $17 \cdot 6$ & $8 \cdot \overline{8}$ & - \\
\hline Trans fatty acids $(\mathrm{g} / \mathrm{d})$ & - & 0.5 & 0.4 & - & $1 \cdot 0$ & $1 \cdot 1$ & - & - & $0 \cdot 4^{*}$ & 0.3 & - & $1 \cdot 0$ & $1 \cdot 3$ & - \\
\hline Cholesterol $(\mathrm{mg} / \mathrm{d})$ & - & $240 \cdot 7$ & $188 \cdot 3$ & - & $271 \cdot 8$ & $194 \cdot 6$ & - & - & $203 \cdot 8$ & $85 \cdot 1$ & - & 249.5 & $211 \cdot 0$ & - \\
\hline Fibre $(g / d) \dagger$ & 31 & $8 \cdot 7$ & $3 \cdot 2$ & 100 & $9 \cdot 6$ & 4.9 & 100 & 26 & $10 \cdot 1$ & $4 \cdot 4$ & 100 & $9 \cdot 6$ & $4 \cdot 2$ & 100 \\
\hline Vitamin $A(\mu \mathrm{g} / \mathrm{d})$ & 600 & $236 \cdot 7$ & $117 \cdot 4$ & 100 & $318 \cdot 1$ & $199 \cdot 9$ & $90 \cdot 4$ & 600 & $290 \cdot 0$ & $170 \cdot 1$ & 100 & $311 \cdot 0$ & $199 \cdot 8$ & $90 \cdot 1$ \\
\hline Vitamin C (mg/d) & 45 & $75 \cdot 4$ & $44 \cdot 4$ & $22 \cdot 2$ & $59 \cdot 4$ & $49 \cdot 0$ & $51 \cdot 5$ & 45 & $98 \cdot 1$ & $58 \cdot 8$ & $16 \cdot 7$ & $71 \cdot 2$ & $62 \cdot 8$ & $43 \cdot 7$ \\
\hline Vitamin D $(\mu \mathrm{g} / \mathrm{d})$ & 15 & 3.5 & $2 \cdot 0$ & 100 & $4 \cdot 4$ & $2 \cdot 0$ & $89 \cdot 0$ & 15 & $4 \cdot 0$ & 1.5 & 100 & 3.5 & $1 \cdot 8$ & 100 \\
\hline Vitamin E (mg/d) & 11 & $2 \cdot 0$ & 0.9 & 100 & $3 \cdot 0$ & $2 \cdot 6$ & $97 \cdot 3$ & 11 & $3 \cdot 0$ & $1 \cdot 1$ & 100 & $2 \cdot 7$ & $1 \cdot 7$ & 100 \\
\hline Vitamin $\mathrm{K}(\mu \mathrm{g} / \mathrm{d}) \dagger$ & 60 & $4 \overline{1} \cdot 0$ & $59 \cdot 6$ & $77 \cdot 8$ & $19 \cdot 4$ & $29 \cdot 9$ & $93 \cdot 2$ & 60 & $24 \cdot 0$ & $20 \cdot 2$ & 100 & $32 \cdot 8$ & $44 \cdot 9$ & $83 \cdot 1$ \\
\hline Thiamin $(\mathrm{mg} / \mathrm{d})$ & 0.9 & 1.0 & 0.4 & $44 \cdot 4$ & $1 \cdot 4$ & 0.7 & $23 \cdot 3$ & 0.9 & $1 \cdot 2$ & 0.5 & $33 \cdot 3$ & $1 \cdot 1$ & 0.5 & $43 \cdot 7$ \\
\hline Riboflavin (mg/d) & 0.9 & $1 \cdot 2$ & 0.3 & $22 \cdot 2$ & 1.5 & 0.7 & $17 \cdot 8$ & 0.9 & 1.5 & 0.6 & $16 \cdot 7$ & $1 \cdot 2$ & 0.5 & $36 \cdot 6$ \\
\hline Niacin $(\mathrm{mg} / \mathrm{d})$ & 12 & $17 \cdot \overline{4}$ & $9 \cdot 2$ & $33 \cdot \overline{3}$ & $18 \cdot 9$ & $10 \cdot 4$ & $27 \cdot 4$ & 12 & $20 \cdot 8$ & $8 \cdot 4$ & $16 \cdot 7$ & $17 \cdot 1$ & $9 \cdot 3$ & $29 \cdot 6$ \\
\hline Vitamin $B_{6}(\mathrm{mg} / \mathrm{d})$ & 1 & $1 \cdot 2$ & 0.3 & $44 \cdot 4$ & $1 \cdot 4$ & 0.7 & $34 \cdot 2$ & 1 & 1.5 & 0.3 & 25 & $1 \cdot 2$ & 0.6 & $33 \cdot 8$ \\
\hline Folate $(\mu \mathrm{g} / \mathrm{d})$ & 300 & $270 \cdot 0$ & $75 \cdot 9$ & $66 \cdot 7$ & $289 \cdot 8$ & $142 \cdot 2$ & $57 \cdot 5$ & 300 & $269 \cdot 7$ & $99 \cdot 1$ & 50 & $261 \cdot 1$ & $133 \cdot 7$ & $64 \cdot 8$ \\
\hline Vitamin $B_{12}(\mu \mathrm{g} / \mathrm{d})$ & $1 \cdot 8$ & $3 \cdot 2$ & 1.5 & $11 \cdot 1$ & $4 \cdot 1$ & $2 \cdot 4$ & $12 \cdot 3$ & $1 \cdot 8$ & $4 \cdot 1$ & 3.9 & $16 \cdot 6$ & $3 \cdot 7$ & $5 \cdot 2$ & $29 \cdot 6$ \\
\hline $\mathrm{Ca}(\mathrm{mg} / \mathrm{d})$ & 1300 & $674 \cdot 0$ & $262 \cdot 3$ & 100 & $767 \cdot 6$ & 335.2 & $95 \cdot 9$ & 1300 & $606 \cdot 2$ & $203 \cdot 1$ & 100 & $585 \cdot 2$ & $276 \cdot 4$ & $98 \cdot 6$ \\
\hline $\mathrm{Cu}(\mathrm{mg} / \mathrm{d})$ & 0.7 & 0.5 & 0.1 & 100 & 0.6 & 0.2 & $69 \cdot 9$ & 0.7 & 0.5 & 0.1 & $91 \cdot 7$ & 0.5 & 0.2 & $73 \cdot 2$ \\
\hline Fluoride $(\mathrm{mg} / \mathrm{d}) \dagger$ & 2 & $0 \cdot 3^{*}$ & $0 \cdot 1$ & 100 & $97 \cdot 7$ & $194 \cdot 0$ & $75 \cdot 3$ & 2 & 0.3 & $0 \cdot 1$ & 100 & 85.0 & $189 \cdot 7$ & $77 \cdot 5$ \\
\hline lodine $(\mu \mathrm{g} / \mathrm{d})$ & 120 & $74 \cdot 7$ & $49 \cdot 0$ & $66 \cdot 7$ & $90 \cdot 5$ & $48 \cdot 7$ & $78 \cdot 1$ & 120 & $88 \cdot 9$ & $35 \cdot 6$ & 75 & $68 \cdot 4$ & $39 \cdot 0$ & $85 \cdot 9$ \\
\hline $\mathrm{Fe}(\mathrm{mg} / \mathrm{d})$ & 8 & $10 \cdot 9$ & $3 \cdot 6$ & $11 \cdot 1$ & $13 \cdot 0$ & $5 \cdot 8$ & $17 \cdot 8$ & 8 & $12 \cdot 6$ & $6 \cdot 4$ & 25 & $11 \cdot 0$ & 4.9 & 31 \\
\hline $\mathrm{Mg}(\mathrm{mg} / \mathrm{d})$ & 240 & $137 \cdot 4$ & $42 \cdot 2$ & 100 & $145 \cdot 3$ & $51 \cdot 3$ & $93 \cdot 2$ & 240 & $148 \cdot 3$ & $37 \cdot 7$ & 100 & $133 \cdot 2$ & $55 \cdot 1$ & $94 \cdot 4$ \\
\hline$P(\mathrm{mg} / \mathrm{d})$ & 1250 & $791 \cdot 3$ & $216 \cdot 9$ & 100 & $876 \cdot 3$ & $297 \cdot 1$ & $89 \cdot 0$ & 1250 & $859 \cdot 6$ & $226 \cdot 1$ & $91 \cdot 7$ & $736 \cdot 3$ & $296 \cdot 9$ & $94 \cdot 4$ \\
\hline $\mathrm{Zn}(\mathrm{mg} / \mathrm{d})$ & 8 & $7 \cdot 4$ & 3.0 & $66 \cdot 7$ & $9 \cdot 1$ & 5.2 & $50 \cdot 7$ & 8 & $7 \cdot 6$ & 4.4 & $66 \cdot 7$ & $7 \cdot 2$ & 3.6 & $66 \cdot 2$ \\
\hline $\mathrm{K}(\mathrm{mg} / \mathrm{d}) \dagger$ & 4500 & $1578 \cdot 6$ & $613 \cdot 0$ & 100 & $1639 \cdot 8$ & $637 \cdot 0$ & 100 & 4500 & $1667 \cdot 8$ & $504 \cdot 2$ & 100 & $1431 \cdot 8$ & $643 \cdot 2$ & 100 \\
\hline $\mathrm{Na}(\mathrm{mg} / \mathrm{d})+$ & 1500 & $1553 \cdot 5^{\star}$ & $398 \cdot 2$ & $33 \cdot 3$ & $2319 \cdot 4$ & $1253 \cdot 6$ & $28 \cdot 8$ & 1500 & $1557 \cdot 6^{*}$ & $585 \cdot 4$ & 75 & $1907 \cdot 3$ & $822 \cdot 6$ & $40 \cdot 8$ \\
\hline
\end{tabular}

DRI refer to the Dietary Reference Intakes ${ }^{(27)}$, including Recommended Dietary Allowances and Adequate Intakes (dashes indicate that values have not been determined).

PBD (percentage below DRI): for a specific nutrient, PBD refers to the percentage of children who consumed a nutrient below the DRI.
${ }^{*}$ Within a specific age group and gender, this superscript indicates significant differences in nutrient consumption between children born in the USA $v$. those born in Thailand/Laos (T/L; $\left.P<0 \cdot 05\right)$.

tAdequate Intakes. 
Table 3 Dietary intakes by birth location for 14-18-year-old Hmong children from Twin Cities, Minnesota, USA, compared with DRI

\begin{tabular}{|c|c|c|c|c|c|c|c|c|c|c|c|c|c|c|}
\hline & \multicolumn{7}{|c|}{ Males (14-18 years; $n$ 68) } & \multicolumn{7}{|c|}{ Females (14-18 years; $n$ 102) } \\
\hline & \multirow[b]{2}{*}{ DRI } & \multicolumn{3}{|c|}{ Born-T/L } & \multicolumn{3}{|c|}{ Born-US } & \multirow[b]{2}{*}{ DRI } & \multicolumn{3}{|c|}{ Born-T/L } & \multicolumn{3}{|c|}{ Born-US } \\
\hline & & Mean & SD & PBD & Mean & SD & PBD & & Mean & SD & PBD & Mean & SD & PBD \\
\hline Acculturation score & - & $25 \cdot 4^{*}$ & $3 \cdot 4$ & - & $29 \cdot 8$ & $4 \cdot 3$ & - & - & $23 \cdot 8^{\star}$ & $3 \cdot 7$ & - & $30 \cdot 6$ & 3.9 & - \\
\hline Energy $(\mathrm{kJ} / \mathrm{d})$ & - & $6338^{\star}$ & 1087 & - & 8732 & 3558 & - & - & 6608 & 2326 & - & 7718 & 3379 & - \\
\hline Energy (kcal/d) & - & $1514 \cdot 7^{\star}$ & $259 \cdot 7$ & - & $2087 \cdot 1$ & $850 \cdot 5$ & - & - & $1579 \cdot 4$ & $555 \cdot 9$ & - & $1844 \cdot 7$ & $807 \cdot 6$ & - \\
\hline Carbohydrates (g/d) & 130 & $181 \cdot 2^{*}$ & $32 \cdot 9$ & 0 & $270 \cdot 6$ & $103 \cdot 9$ & $3 \cdot 3$ & 130 & $198 \cdot 5$ & 74.9 & $33 \cdot 3$ & $233 \cdot 4$ & $100 \cdot 3$ & $14 \cdot 6$ \\
\hline Protein $(g / d)$ & 52 & $81 \cdot 5$ & $24 \cdot 0$ & 0 & $90 \cdot 3$ & $49 \cdot 7$ & $10 \cdot 0$ & 46 & $87 \cdot 7$ & $30 \cdot 9$ & $16 \cdot 7$ & $82 \cdot 1$ & $53 \cdot 2$ & $21 \cdot 9$ \\
\hline Fat $(g / d)$ & - & $50 \cdot 6$ & $14 \cdot 6$ & - & $71 \cdot 7$ & $40 \cdot 1$ & - & - & $47 \cdot 0$ & $20 \cdot 2$ & - & $64 \cdot 0$ & $35 \cdot 5$ & - \\
\hline Saturated fat $(g / d)$ & - & $16 \cdot 6$ & $5 \cdot 0$ & - & $23 \cdot 8$ & $12 \cdot 9$ & - & - & $14 \cdot 2$ & $6 \cdot 3$ & - & $20 \cdot 9$ & $12 \cdot 3$ & - \\
\hline Trans fatty acids $(\mathrm{g} / \mathrm{d})$ & - & 0.7 & $0 \cdot 6$ & - & $1 \cdot 3$ & $1 \cdot 0$ & - & - & $0 \cdot 6^{\star}$ & $0 \cdot 4^{*}$ & - & $1 \cdot 3$ & $1 \cdot 6$ & - \\
\hline Cholesterol $(\mathrm{mg} / \mathrm{d})$ & - & $223 \cdot 6$ & $94 \cdot 3$ & - & $322 \cdot 4$ & $232 \cdot 6$ & - & - & $280 \cdot 9^{*}$ & $51 \cdot 7$ & - & $260 \cdot 9$ & $265 \cdot 7$ & - \\
\hline Fibre $(g / d) \dagger$ & 38 & $8 \cdot 7$ & $3 \cdot 3$ & $87 \cdot 5$ & $11 \cdot 4$ & $5 \cdot 7$ & 100 & 26 & $9 \cdot 1$ & 4.9 & 100 & $11 \cdot 4$ & $7 \cdot 1$ & $94 \cdot 8$ \\
\hline Vitamin A ( $\mu \mathrm{g} / \mathrm{d})$ & 900 & $348 \cdot 1$ & $340 \cdot 8$ & $75 \cdot 0$ & $376 \cdot 5$ & $249 \cdot 2$ & $96 \cdot 7$ & 700 & $278 \cdot 0$ & $175 \cdot 1$ & 100 & $293 \cdot 3$ & $299 \cdot 0$ & $92 \cdot 7$ \\
\hline Vitamin C (mg/d) & 75 & $46 \cdot 0^{\star}$ & $26 \cdot 0$ & $50 \cdot 0$ & $89 \cdot 7$ & $90 \cdot 0$ & $43 \cdot 3$ & 65 & $43 \cdot 1$ & $35 \cdot 2$ & $66 \cdot 7$ & $88 \cdot 2$ & $99 \cdot 5$ & $47 \cdot 9$ \\
\hline Vitamin $D(\mu \mathrm{g} / \mathrm{d})$ & 15 & $4 \cdot 1$ & $1 \cdot 9$ & 100 & $4 \cdot 6$ & $3 \cdot 1$ & $98 \cdot 3$ & 15 & $3 \cdot 6$ & $1 \cdot 6$ & 100 & $3 \cdot 0$ & $2 \cdot 3$ & $94 \cdot 8$ \\
\hline Vitamin E (mg/d) & 15 & $2 \cdot 1^{*}$ & $1 \cdot 1$ & $87 \cdot 5$ & $4 \cdot 1$ & $3 \cdot 5$ & $98 \cdot 3$ & 15 & $2 \cdot 2$ & $1 \cdot 1$ & 100 & $3 \cdot 9$ & $3 \cdot 9$ & $97 \cdot 9$ \\
\hline Vitamin $K(\mu \mathrm{g} / \mathrm{d}) \dagger$ & 75 & $45 \cdot 5$ & $39 \cdot 4$ & $37 \cdot 5$ & $34 \cdot 1$ & $54 \cdot 9$ & $85 \cdot 0$ & 75 & $16 \cdot 4$ & $10 \cdot 9$ & 100 & $45 \cdot 9$ & $68 \cdot 5$ & $80 \cdot 2$ \\
\hline Thiamin $(\mathrm{mg} / \mathrm{d})$ & $1 \cdot 2$ & $1 \cdot 6$ & $1 \cdot 1$ & $37 \cdot 5$ & 1.5 & $0 \cdot 7$ & $20 \cdot 0$ & $1 \cdot 0$ & $1 \cdot 6$ & $1 \cdot 0$ & $33 \cdot 3$ & $1 \cdot 5$ & $0 \cdot 7$ & $34 \cdot 4$ \\
\hline Riboflavin (mg/d) & $1 \cdot 3$ & $1 \cdot 3$ & 0.9 & $62 \cdot 5$ & $1 \cdot 7$ & $1 \cdot 0$ & $21 \cdot 7$ & $1 \cdot 0$ & $1 \cdot 3$ & $0 \cdot 8$ & $33 \cdot 3$ & $1 \cdot 3$ & 0.9 & $42 \cdot 7$ \\
\hline Niacin $(\mathrm{mg} / \mathrm{d})$ & 16 & $18 \cdot 1$ & $10 \cdot 1$ & $50 \cdot 0$ & $24 \cdot 5$ & $15 \cdot 4$ & $23 \cdot 3$ & 14 & $21 \cdot 5$ & $12 \cdot 7$ & $33 \cdot 3$ & $20 \cdot 4$ & $13 \cdot 6$ & $33 \cdot 3$ \\
\hline Vitamin $B_{6}(\mathrm{mg} / \mathrm{d})$ & $1 \cdot 3$ & $1 \cdot 3$ & $0 \cdot 8$ & $37 \cdot 5$ & $1 \cdot 8$ & $1 \cdot 1$ & $16 \cdot 7$ & $1 \cdot 2$ & 1.5 & 0.9 & $33 \cdot 3$ & $1 \cdot 4$ & $1 \cdot 0$ & $34 \cdot 4$ \\
\hline Folate $(\mu \mathrm{g} / \mathrm{d})$ & 400 & $269 \cdot 2$ & 111.5 & $75 \cdot 0$ & $376 \cdot 7$ & $203 \cdot 3$ & $58 \cdot 3$ & 400 & $278 \cdot 2$ & $147 \cdot 3$ & $83 \cdot 3$ & $297 \cdot 0$ & $201 \cdot 9$ & $80 \cdot 2$ \\
\hline Vitamin $B_{12}(\mu \mathrm{g} / \mathrm{d})$ & $2 \cdot 4$ & $4 \cdot 1$ & $2 \cdot 3$ & $12 \cdot 5$ & $4 \cdot 7$ & $3 \cdot 3$ & $21 \cdot 7$ & $2 \cdot 4$ & $3 \cdot 2$ & $2 \cdot 7$ & $37 \cdot 5$ & $4 \cdot 0$ & $4 \cdot 3$ & $36 \cdot 5$ \\
\hline $\mathrm{Ca}(\mathrm{mg} / \mathrm{d})$ & 1300 & $559 \cdot 8$ & $222 \cdot 8$ & $87 \cdot 5$ & $736 \cdot 9$ & $392 \cdot 6$ & $93 \cdot 3$ & 1300 & $454 \cdot 2$ & $252 \cdot 3$ & 100 & $552 \cdot 4$ & $306 \cdot 0$ & $97 \cdot 9$ \\
\hline $\mathrm{Cu}(\mathrm{mg} / \mathrm{d})$ & 0.89 & $0 \cdot 5^{\star}$ & 0.2 & 100 & $0 \cdot 8$ & 0.3 & $56 \cdot 7$ & 0.89 & 0.5 & 0.2 & $83 \cdot 3$ & 0.7 & 0.3 & $77 \cdot 1$ \\
\hline Fluoride $(\mathrm{mg} / \mathrm{d}) \dagger$ & 3 & $329 \cdot 7^{\star}$ & $124 \cdot 5$ & 0 & $185 \cdot 4$ & $323 \cdot 9$ & $68 \cdot 3$ & 3 & $280 \cdot 8$ & $244 \cdot 2$ & $33 \cdot 3$ & $160 \cdot 4$ & $239 \cdot 1$ & $61 \cdot 5$ \\
\hline Iodine $(\mu \mathrm{g} / \mathrm{d})$ & 150 & $69 \cdot 3$ & $36 \cdot 4$ & $87 \cdot 5$ & $88 \cdot 1$ & $64 \cdot 7$ & $83 \cdot 3$ & 150 & $68 \cdot 0$ & $44 \cdot 4$ & 100 & $58 \cdot 3$ & $49 \cdot 5$ & $94 \cdot 8$ \\
\hline $\mathrm{Fe}(\mathrm{mg} / \mathrm{d})$ & 11 & $12 \cdot 7$ & $4 \cdot 3$ & $25 \cdot 0$ & $15 \cdot 0$ & $7 \cdot 5$ & $28 \cdot 3$ & 15 & $12 \cdot 7$ & $6 \cdot 7$ & $66 \cdot 7$ & $13 \cdot 7$ & 8.5 & $68 \cdot 7$ \\
\hline $\mathrm{Mg}(\mathrm{mg} / \mathrm{d})$ & 410 & $137 \cdot 6$ & $50 \cdot 3$ & $87 \cdot 5$ & $184 \cdot 2$ & $79 \cdot 0$ & $98 \cdot 3$ & 360 & $149 \cdot 0$ & $65 \cdot 4$ & 100 & $155 \cdot 8$ & $78 \cdot 3$ & $97 \cdot 9$ \\
\hline$P(\mathrm{mg} / \mathrm{d})$ & 1250 & $843 \cdot 8$ & 343.5 & $75 \cdot 0$ & $1048 \cdot 1$ & $461 \cdot 6$ & 75 & 1250 & $862 \cdot 7$ & $351 \cdot 9$ & $66 \cdot 7$ & $867 \cdot 2$ & $485 \cdot 4$ & $85 \cdot 4$ \\
\hline $\mathrm{Zn}(\mathrm{mg} / \mathrm{d})$ & 11 & $9 \cdot 1$ & $4 \cdot 1$ & $75 \cdot 0$ & $10 \cdot 5$ & $7 \cdot 0$ & $61 \cdot 7$ & 9 & $7 \cdot 0$ & 3.5 & $83 \cdot 3$ & $10 \cdot 8$ & 11.9 & $58 \cdot 3$ \\
\hline $\mathrm{K}(\mathrm{mg} / \mathrm{d}) \dagger$ & 4700 & $1533 \cdot 2$ & $705 \cdot 6$ & $87 \cdot 5$ & $1970 \cdot 0$ & $840 \cdot 5$ & 100 & 4700 & $1520 \cdot 5$ & $657 \cdot 8$ & 100 & $1666 \cdot 4$ & $910 \cdot 4$ & 98.9 \\
\hline $\mathrm{Na}(\mathrm{mg} / \mathrm{d})+$ & 1500 & $1945 \cdot 8^{*}$ & $693 \cdot 3$ & $25 \cdot 0$ & $2901 \cdot 01$ & $444 \cdot 8$ & $15 \cdot 0$ & 1500 & $1424 \cdot 9^{*}$ & $559 \cdot 4$ & $50 \cdot 0$ & $2426 \cdot 8$ & $1381 \cdot 9$ & $25 \cdot 0$ \\
\hline
\end{tabular}

DRI refer to the Dietary Reference Intakes ${ }^{(27)}$, including Recommended Dietary Allowances and Adequate Intakes (dashes indicate that values have not been determined).

PBD (percentage below DRI): for a specific nutrient, PBD refers to the percentage of children who consumed a nutrient below the DRI.

"Within a specific age group and gender, this superscript indicates significant differences in nutrient consumption between children born in the USA $v$. those born in Thailand/Laos (T/L; $P<0.05)$.

tAdequate Intakes. 
The results from $24 \mathrm{~h}$ data and MyPyramid analysis were then imported into PASW (version 17) for further analysis. Per MyPyramid guidelines ${ }^{(30)}$, children $<12$ years of age have lower serving suggestions for fruits and meat and beans than older children, and were therefore compared separately (Table 4). The independent-samples $t$ test was used to compare differences in nutrient and food group intakes between children born-US $v$. born-T/L. The associations among years lived in the USA, acculturation scores, BMI and nutrients consumed were calculated by Pearson correlation $(r)$. The significance level was set at $P<0 \cdot 05$.

\section{Results}

\section{Sample characteristics}

Sample characteristics of Hmong children are shown in Table 1 . Mean age of participants was $13 \cdot 6$ (SD 2.6) years. Some $45 \cdot 1 \%$ attended high school, $33 \cdot 7 \%$ middle school and $20.6 \%$ elementary schools. Sixteen per cent of the children were overweight (BMI-for-age $\geq 85$ th to $<95$ th percentile) and $30 \%$ were obese (BMI-for-age $\geq 95$ th percentile) for their ages. Further, 23.3\% were short statured (height-for-age $<5$ th percentile) and $4.5 \%$ were tall for their ages (height-for-age $\geq 85$ th percentile). Compared with born-T/L, born-US children were significantly heavier (mean (SD): $55 \cdot 8(18 \cdot 1) \mathrm{kg} v .44 \cdot 2(11 \cdot 2)$ $\mathrm{kg}$ ), taller (mean (sD): $151 \cdot 3(10 \cdot 9) \mathrm{cm} v .146 \cdot 9(11 \cdot 1) \mathrm{cm}$ ) and had a higher BMI (mean (SD): $23 \cdot 9(5 \cdot 8) \mathrm{kg} / \mathrm{m}^{2} v \cdot 20 \cdot 2$ (3.0) $\mathrm{kg} / \mathrm{m}^{2} ; P<0 \cdot 05$ for all comparisons).

\section{$24 \mathrm{~b}$ dietary recall}

The $24 \mathrm{~h}$ dietary recall results are shown in Tables 2 and 3. In general, diets of most children were below the DRI levels for fibre, vitamins $\mathrm{A}, \mathrm{D}$ and $\mathrm{E}, \mathrm{Ca}, \mathrm{Mg}$ and $\mathrm{K}$. Among 9-13 year-old males, born-US consumed significantly more energy, carbohydrates, fat, saturated fat, $\mathrm{Na}$ and fluoride than born-T/L ones $(P<0 \cdot 05)$. Among 9-13-year-old females, born-US consumed higher amounts of trans fatty acids and $\mathrm{Na}$ than their born-T/L counterparts $(P<0 \cdot 05$; Table 2$)$. Approximately one-third of 9-13-year-old females did not meet DRI recommendations for Fe. Among 14-18-year-old males, born-US consumed more energy, carbohydrates, vitamins $\mathrm{C}$ and $\mathrm{E}$, $\mathrm{Cu}, \mathrm{Na}$ and fluoride than born-T/L ones $(P<0 \cdot 05)$. Further, among 14-18-year-old females, those born-US consumed more trans fatty acids and $\mathrm{Na}$, and less cholesterol, than their born-T/L counterparts $(P<0 \cdot 05$; Table 3$)$. About two-thirds of 14-18-year-old females did not meet DRI recommendations for $\mathrm{Fe}$.

\section{MyPyramid analysis}

MyPyramid analysis indicated that most 9-11-year-olds consumed less vegetables and milk than the suggested servings (Table 4). Further, the majority of 12-18-year-olds

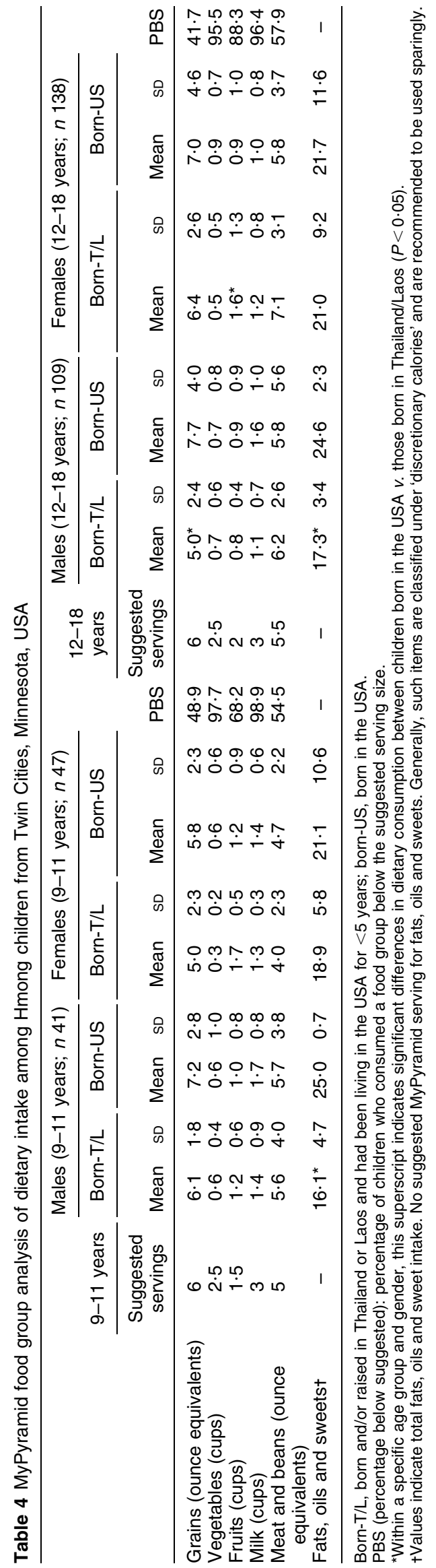


consumed less fruits, vegetables and milk than recommended. Born-US males consumed significantly higher amounts of fats, oils and sweets than born-T/L males $(P<0 \cdot 05)$, while no significant differences were observed among females in this regard (Table 4). Among 12-18year-old females, born-US consumed less fruits than the born-T/L ones $(P=0 \cdot 04)$. Among 12-18-year-old males, born-US consumed more grains than the born-T/L ones $(P=0 \cdot 01)$.

\section{Dietary associations}

Among all children, acculturation was positively associated with consumption of carbohydrates $(r=0 \cdot 12$, $P=0.03)$, saturated fat $(r=0.13, P=0 \cdot 02)$, trans fatty acids $(r=0 \cdot 13, P=0 \cdot 02), \mathrm{Ca}(r=0 \cdot 16, P=0 \cdot 005), \mathrm{Na}$ $(r=0 \cdot 21, P<0 \cdot 001)$, and fats, oils and sweets $(r=0 \cdot 11$, $P=0 \cdot 04)$. Additionally, more acculturated children had a higher BMI-for-age compared with less acculturated ones ( $r=0 \cdot 16, P=0 \cdot 005)$. Likewise, the number of years lived in the USA was positively associated with consumption of energy $(r=0 \cdot 23, P<0 \cdot 001)$, saturated fat $(r=0 \cdot 18$, $P=0 \cdot 001)$, trans fatty acids $(r=0 \cdot 17, P=0 \cdot 003)$, fibre $(r=0 \cdot 13, P=0 \cdot 02), \mathrm{Na}(r=0 \cdot 29, P<0 \cdot 001)$, fats, oils and sweets $(r=0 \cdot 17, P=0 \cdot 002)$ and BMI-for-age $(r=0 \cdot 39$, $P<0 \cdot 001)$. Higher BMI was also associated with a significantly higher consumption of $\mathrm{Na}(r=0 \cdot 16, P=0 \cdot 004)$, and a significantly lower consumption of fruits $(r=-0 \cdot 21$, $P<0 \cdot 001)$ and milk $(r=-0 \cdot 16, P=0 \cdot 004)$.

\section{Discussion}

The results of our study suggest that diets of Hmong children are low in nutrients such as $\mathrm{Ca}, \mathrm{Fe}$, vitamins $\mathrm{A}$ and $\mathrm{D}, \mathrm{P}$ and fibre, and high in $\mathrm{Na}$ and fats, oils and sweets. This is reflected in low consumption levels of vegetables and milk, and high consumption of energydense foods. In general, US-born children consumed more energy, carbohydrates, saturated fat and $\mathrm{Na}$, and had a higher BMI, than those born in Thailand/Laos (and had been in the USA for $<5$ years), suggesting that an obesogenic US environment is a probable reason for poor dietary habits among Hmong children. Additionally, the $24 \mathrm{~h}$ dietary recalls of most US-born children included items such as muffins, cakes, chips, soda, chocolate milk, pizza, burgers and fried meats, and most US-born children reported using high-Na sauces as added seasonings (results not shown). Most Thailand/Laos-born children consumed boiled meats, cooked vegetables, steamed rice, candy and cookies (results not shown). No significant differences in rice consumption were noted between the two groups (mean (SD): $1 \cdot 2(0 \cdot 92) \mathrm{cups} / \mathrm{d}$ for born-US $v \cdot 1 \cdot 3(0 \cdot 61)$ cups/d for born-T/L).

Children who were more acculturated to US norms including language use, social connections and dietary habits had a higher BMI-for-age compared with their less acculturated counterparts. About half of our participants were either overweight or obese. Research has indicated that obesity during childhood and adolescent years is a risk factor for developing CHD, hypertension, dyslipidaemia, type 2 diabetes, and even results in premature mortality in adulthood ${ }^{(31,32)}$, suggesting that most Hmong children in our sample may be at a risk for developing such conditions in their near future.

\section{Dietary status of Hmong children}

More than $90 \%$ of the children in our sample did not meet the MyPyramid recommendations for the dairy food group including milk, yoghurt and cheese. This observation is similar to national trends in dairy consumption among children, with more than half of children aged 2-8 years and three-quarters of children aged 9-19 years not consuming recommended dairy servings ${ }^{(33)}$. Nutrients such as Ca, vitamin $\mathrm{D}, \mathrm{P}$ and protein are found in the dairy food group and are required to support growth and development during childhood and adolescent years, including reaching peak bone mass. It is believed that about $85-90 \%$ of the final adult bone mass is acquired by the age of $18-20$ years $^{(34)}$, necessitating the inclusion of bone-building nutrients during childhood. Possible reasons for low dairy consumption in our sample may be related to high lactose intolerance found among Asians ${ }^{(35)}$, and not consuming milk because of taste preferences and/or cultural reasons ${ }^{(36)}$. Inadequate intakes of $\mathrm{Ca}$ and vitamin $\mathrm{D}$ during developmental years may increase the risk for osteoporosis later in life ${ }^{(37,38)}$. To decrease future cases of osteoporosis, schools should be encouraged to increase $\mathrm{Ca}$ intake among children either by encouraging milk and yoghurt consumption among non-lactose intolerant children or by providing non-dairy fortified foods such as juices, cereals and grains to those with lactose intolerance.

The mean Fe consumption was below the DRI for approximately $67 \%$ of 14-18-year-old females (mean 13.6 (SD 8.4) $\mathrm{mg}$; DRI $=15 \mathrm{mg} / \mathrm{d}$ ). Similar to our results, the National Health and Nutrition Examination Survey (1999-2000) estimated an average Fe intake of $13.4 \mathrm{mg} / \mathrm{d}$ among females aged $12-19$ years $^{(39)}$. Fe deficiency affects $2 \cdot 4$ million children in the USA, and it is one of the most common nutritional deficiencies among menstruating adolescents and women ${ }^{(40)}$. Fe deficiency limits the delivery of oxygen to cells, resulting in decreased immunity, increased fatigue, poor work performance and, among pregnant women, delivery of low-birthweight infants ${ }^{(41,42)}$. Our results indicated lower consumption levels of Fe among Hmong children and oral supplementation might be a potential source of Fe for this group. Research suggests that long-term oral Fe supplementation can improve cognitive abilities including attention span and the ability to concentrate ${ }^{(43,44)}$.

The diets of most Hmong children were below the recommendations for fibre; the mean fibre intake was 
about $10 \mathrm{~g} / \mathrm{d}$. Further, the mean vegetable intake among all children was less than $1 \mathrm{cup} / \mathrm{d}$. Within the USA, it is estimated that only $39 \%$ of children within the 2-17 years age range meet the US Department of Agriculture's dietary recommendations for fibre ${ }^{(45)}$. As reported in the literature, Hmong-American diets tend to be low in fibrerich foods such as whole grains, fruits and vegetables, partly because of acculturation to US dietary norms ${ }^{(13,46)}$. We found no significant differences in fibre consumption between children consuming traditional diets and those consuming more Americanized diets. Franzen and Smith ${ }^{(4)}$ reported low intake levels of fruits among Hmong because fruits were considered as luxury items and consumed sparingly, often as a dessert. Also, fruits that were preferred and easily available in Thailand/Laos, such as jackfruit, mango, guava, papaya and pineapple, are either hard to find or too expensive to purchase in the USA, further decreasing fruit consumption ${ }^{(13)}$. While a diet rich in fibre has many health benefits such as lowering LDL cholesterol, decreasing the incidence of CVD and diabetes, preventing obesity, limiting total energy intake and providing other important micronutrients ${ }^{(47)}$, it will be a challenge to health-care professionals to create ways to increase fibre in this Asian subgroup. Our results suggest that Hmong children would benefit from early education about the benefits of fibre and foods rich in fibre, with emphasis on the consumption of whole grains, fruits and vegetables. This might be best accomplished at school through the National School Lunch Program by including whole grain food choices, fresh fruits and salads on the school menu. Further, involving parents in educational and/or physical activity programmes with their children could improve activity levels, although this has not been evaluated among Hmong. Parental participation will be important because Hmong parents (specifically the recently immigrated ones) might perceive losing weight as a negative health condition, because being heavy is generally perceived as being beautiful and healthy in traditional Hmong culture ${ }^{(48)}$. One such intervention could be incorporating gardening projects in school curricula/community programmes, with parents and children planting seasonal fruits and vegetables as a family.

\section{Acculturation and dietary intake}

Number of years lived in the USA and acculturation to US dietary habits were associated with a higher consumption of energy, trans fatty acids, saturated fats, sugars and $\mathrm{Na}$, and may partly explain why about half of our sample was overweight/obese. In a sample of low-income Puerto Rican women living in the USA, Himmelgreen et al. ${ }^{(49)}$ found significant increases in BMI with the length of time stayed in the USA. Research also indicates that after immigrating and acculturating to the US environment, sedentary habits, busy lifestyle and physical inactivity have led to increases in overweight and obesity in the Hmong population ${ }^{(4,5,13)}$. Stang et al. $^{(14)}$ found that when compared with white adolescents, Hmong adolescents reported less physical activity and were at an increased risk for obesity. Most Hmong migrated from areas where they worked hard in farm fields; manual labour was the primary economic source. Post-migration, many Hmong adopt a sedentary lifestyle, and have less time for being physically active ${ }^{(4,50)}$. Further, many still associate physical activity with occupation and are usually not interested in exercising during leisure time ${ }^{(4)}$. Further, Asian Americans appear to be genetically susceptible to develop abdominal obesity and insulin resistance and the risk of type 2 diabetes among Asians starts at a lower $\mathrm{BMI}^{(51)}$, emphasizing the importance of a healthy diet and physical activity among Hmong from an earlier age.

Results from the present study also indicate that children who were born in the USA consumed significantly more $\mathrm{Na}$ than their Thailand/Laos-born counterparts. Research has shown that number of years lived in the USA and acculturation to US dietary patterns are associated with increased $\mathrm{Na}$ consumption and consequently a higher prevalence of hypertension among immigrant populations ${ }^{(52)}$. Because the children in our study are in their preadolescent to adolescent years, consuming high-Na diets makes them susceptible to develop hypertension and associated conditions such as CVD if measures to educate them about healthy lifestyle are not taken soon.

\section{Limitations and conclusions}

Although our study is the first one to demonstrate a detailed, descriptive quantitative analysis of Hmong diets from an acculturation perspective, nevertheless it has some limitations. Some participants may have under/ over-reported their food intake. Earlier research has found that overweight/obese respondents, women and weight-conscious people tend to under-report their food intake because of social desirability, probably leading to respondent bias during data collection ${ }^{(20,53,54)}$. Second, interviewer bias is a common form of error within $24 \mathrm{~h}$ dietary recalls ${ }^{(20)}$, and some participant dietary information might have been missed, misunderstood or incorrectly recorded by the researchers. However, we believe that using the multiple-pass interview technique and incorporating memory prompts such as food models and measuring cups/spoons during the interviews minimized this problem. Third, the born-T/L sample was smaller in size than the born-US one, making the comparisons between these two groups somewhat difficult. However, statistical tests including $t$ tests adjust for the sample size and some results were found to be significant while comparing born-US children with the born-T/L ones. Finally, although we recruited a representative sample of Hmong children in Minnesota, our results cannot be generalized to all Hmong children living in the USA. Given that the Hmong are a fast-growing Asian ethnic 
subgroup in the $\mathrm{USA}^{(2,55)}$, it is important to learn more about their nutritional status and needs from a health-care perspective. Our findings indicate high intakes of fats, sweets and $\mathrm{Na}$ among young Hmong and suggest a need for dietary education and intervention among Hmong children towards eating healthier foods.

\section{Acknowledgements}

The present study was funded by the University of Minnesota Agricultural Experiment Station. No author has a conflict of interest. This manuscript is an original contribution and has not been published elsewhere; all authors collected data and contributed to the interpretation of the data, the writing of the manuscript, and read and approved this version of the manuscript. U.M.-P. collected, entered and analysed data, and took the lead in the writing of the manuscript. C.S., project Principal Investigator, conceptualized the design of the project, collected data, supervised the data analyses, and assisted in the writing and editing of the manuscript. L.F.-C. collected, entered and analysed the data, and read through and edited the manuscript.

\section{References}

1. US Census Bureau (2007) Minority population tops 100 million. http://www.census.gov/newsroom/releases/archives/ population/cb07-70.html (accessed March 2011).

2. US Census Bureau (2011) Asian/Pacific American Heritage Month: May 2011. http://www.census.gov/newsroom/releases/ archives/facts_for_features_special_editions/cb11-ff06.html (accessed January 2011).

3. Yang K (2001) The Hmong in America: twenty-five years after the US secret war in Laos. J Asian Am Stud 4, 165-174.

4. Franzen L \& Smith C (2009) Acculturation and environmental change impacts dietary habits among adult Hmong. Appetite 52, 173-183.

5. Franzen L \& Smith C (2009) Differences in stature, BMI, and dietary practices between US born and newly immigrated Hmong children. Soc Sci Med 29, 442-450.

6. Fadiman A (1997) The Spirit Catches You and You Fall Down: A Hmong Child, Her American Doctors, and the Collision of Two Cultures. New York: The Noonday Press.

7. Johnson SK (2002) Hmong health beliefs and experiences in the western health care system. J Transcult Nurs 13, 126-132.

8. Lee TP \& Pfeifer ME (2007) Building bridges: teaching about the Hmong in our communities. http://www.hmongcc.org/ BuildingBridgesHealthPresentation2007Version.pdf (accessed January 2011).

9. Pfeifer ME \& Lee S (2004) Hmong population, demographic, socioeconomic, and educational trends in the 2000 Census. Hmong 2000 Census Publication: data and analysis. A publication of Hmong National Development, Inc. and the Hmong Cultural and Resource Center. http:// hmongstudies.org/2000HmongCensusPublication.pdf (accessed March 2011).

10. Clarkin PF (2008) Adiposity and height of adult Hmong refugees: relationship with war-related early malnutrition and later migration. Am J Hum Biol 20, 174-184.

11. Franzen L \& Smith C (2010) Food system access, shopping behavior, and influences on purchasing groceries in adult
Hmong living in Minnesota. Am J Health Promot 24, 396-409.

12. Culhane-Pera KA, Moua M, DeFor T et al. (2009) Cardiovascular disease risks in Hmong refugees from Wat Tham Krabok, Thailand. J Immigr Minor Health 11, 372-379.

13. Goody CM \& Drago L (2010) Cultural Food Practices. Chicago, IL: American Dietetic Association.

14. Stang J, Kong A, Story M et al. (2007) Food and weightrelated patterns and behaviors of Hmong adolescents. J Am Diet Assoc 107, 936-941.

15. McCarty DJ (2005) Glucose intolerance in Wisconsin's Hmong population. Wis Med J 104, 13-14.

16. Yang A, Xiong D, Vang E et al. (2009) Hmong American women living with diabetes. J Nurs Scholarsh 41, 139-148.

17. Her C \& Mundt M (2005) Risk prevalence for type 2 diabetes mellitus in adult Hmong in Wisconsin: a pilot study. Wis Med J 104, 70-77.

18. Centers for Disease Control and Prevention (2008) National Diabetes Factsheet, 2007. http://www.cdc.gov/diabetes/ pubs/pdf/ndfs_2007.pdf (accessed February 2011).

19. Vue H \& Reicks M (2007) Individual and environmental influences on intake of calcium-rich food and beverages by young Hmong adolescent girls. J Nutr Educ Behav 39, 264-272.

20. Gibson RS (2005) Principles of Nutritional Assessment, 2nd ed. New York: Oxford University Press.

21. Committee on Food Consumption Patterns, Food and Nutrition Board, National Research Council (1981) Assessing Changing Food Consumption Patterns. Washington, DC: National Academy Press.

22. McPherson RS, Hoelscher DM, Alexander M et al. (2000) Dietary assessment methods among school-aged children: validity and reliability. Prev Med 31, issue 2, S11-S33.

23. Frank GC (1991) Taking a bite out of eating behavior: food records and food recalls of children. J Sch Health 61, 198-200.

24. Marin G, Sabogal F, Marin BV et al. (1987) Development of a short acculturation scale for Hispanics. Hispanic J Behav Sci 9, 183-205.

25. Frisancho AR (2008) Anthropometric Standards: An Interactive Nutritional Reference of Body Size and Body Composition for Children and Adults. Ann Arbor, MI: University of Michigan Press.

26. Centers for Disease Control and Prevention (2009) About BMI for children and teens. http://www.cdc.gov/ healthyweight/assessing/bmi/childrens_BMI/about_childrens_ BMI.html (accessed December 2010).

27. Dietary Reference Intakes (DRIs): Recommended Dietary Allowances and Adequate Intakes (2010) Food and Nutrition Board, Institute of Medicine, National Academies. http:// www.iom.edu (accessed January 2011).

28. US Department of Agriculture (1992) The Food Guide Pyramid. Home and Garden Bulletin no. 252. Hyattsville, MD: USDA.

29. ESHA Research (2010) How pyramid values are calculated: Calculating food guide pyramid values at ESHA Research. http://www.esha.com/pyramidscalculated (accessed January 2011).

30. US Department of Agriculture (2010) MyPyramid.gov for Kids. http://www.mypyramid.gov/kids/index.html (accessed December 2010).

31. Bibbins-Domingo K, Coxson P, Pletcher MJ et al. (2007) Adolescent overweight and future adult coronary heart disease. $N$ Engl J Med 357, 2371-2379.

32. Engeland A, Bjorge T, Tverdal A et al. (2004) Obesity in adolescence and adulthood and the risk of adult mortality. Epidemiology 15, 79-85.

33. National Dairy Council (2009) Child nutrition: a focus on dairy foods. http://www.nationaldairycouncil.org/ SiteCollectionDocuments/child_nutrition/general_nutrition/ dcd802.pdf (accessed March 2011). 
34. Heaney RP, Abrams S, Dawson-Hughes B et al. (2000) Peak bone mass. Osteoporos Int 11, 985-1009.

35. National Institutes of Health (2009) Lactose Intolerance. http:// digestive.niddk.nih.gov/ddiseases/pubs/lactoseintolerance/ lactoseintolerance.pdf (accessed February 2011).

36. Whitney E \& Rolfes SR (2008) Understanding Nutrition with Environmental Science. Belmont, CA: Thomson Wadsworth.

37. Kirby M \& Danner E (2009) Nutritional deficiencies in children on restricted diets. Pediatr Clin $N$ Am 56, 1085-1103.

38. Tan S, Ji L, Tsai J et al. (2009) Greater osteoporosis educational outreach is desirable among Chinese immigrants in Chinatown, Chicago. Osteoporos Int 20, 1517-1522.

39. Wright JD, Wang CY, Kennedy-Stephenson J et al. (2003) Dietary Intake of Ten Key Nutrients for Public Health, United States: 1999-2000. Advance Data from Vital and Health Statistics no. 334. Hyattsville, MD: National Center for Health Statistics.

40. Brotanek JM, Gosz J, Weitzman M et al. (2007) Iron deficiency in early childhood in the United States: risk factors and racial/ethnic disparities. Pediatrics 120 $568-575$.

41. Kirchengast S \& Hartmann B (1998) Maternal prepregnancy weight status and pregnancy weight gain as major determinants for newborn weight and size. Ann Hum Biol 25, 17-28.

42. Grondin MA, Ruivard M, Perreve A et al. (2008) Prevalence of iron deficiency and health-related quality of life among female students. J Am Coll Nutr 27, 337-341.

43. Haas JD \& Brownlie T (2001) Iron deficiency and reduced work capacity: a critical review of the research to determine a causal relationship. J Nutr 131, 2 Suppl. 2, 691S-696S

44. Falkingham M, Abdelhamid A, Curtis P et al. (2010) The effects of oral iron supplementation on cognition in older children and adults: a systematic review and meta-analysis. Nutr J 9, 4
45. Centers for Disease Control and Prevention (2008) Nutrition and the health of young people. http://www.cdc.gov/ HealthyYouth/nutrition/pdf/facts.pdf (accessed March 2011).

46. Yang RC \& Mills PK (2008) Dietary and lifestyle practices of Hmong in California. J Health Care Poor Underserved 19, 1258-1269.

47. Marlett JA \& Slavin JL (1997) Position of the American Dietetic Association: health implications of dietary fiber. J Am Diet Assoc 97, 1157-1159.

48. Mulasi-Pokhriyal U \& Smith C (2010) Assessing body image issues and body satisfaction/dissatisfaction among Hmong American children 9-18 years of age using mixed methodology. Body Image 7, 341-348.

49. Himmelgreen DA, Perez-Escamilla R, Martinez D et al. (2004) The longer you stay, the bigger you get: length of time and language use in the US are associated with obesity in Puerto Rican women. Am J Phys Anthropol 125, 90-96.

50. Kim LP, Harrison GG \& Kagawa-Singer M (2007) Perceptions of diet and physical activity among California Hmong adults and youth. Prev Chronic Dis 4 , A93.

51. Chan JCN, Malik V, Jia W et al. (2009) Diabetes in Asia: epidemiology, risk factors, and pathophysiology. J Am Diet Assoc 301, 2129-2140.

52. Moran A, Roux AVD, Jackson SA et al. (2007) Acculturation is associated with hypertension in a multiethnic sample. Am J Hypertens 20, 354-363.

53. Harnack LJ, Jeffery RW \& Boutelle KN (2000) Temporal trends in energy intake in the United States: an ecologic perspective. Am J Clin Nutr 71, 1478-1484.

54. Briefel RR, Sempos CT, McDowell MA et al. (1997) Dietary methods research in the third National Health and Nutrition Examination Survey: underreporting of energy intake. Am J Clin Nutr 65, 4 Suppl., 1203S-1209S.

55. Pfeifer ME (2003) Hmong Americans. Asian-Nation: The Landscape of Asian America. http://www.asian-nation.org/ hmong.shtml (accessed January 2011). 